\title{
MULTILINGUALISM AND LINGUISTIC CAPACITY BUILDING
}

\section{BINYAM SISAY MENDISU \& JANNE BONDI JOHANNESSEN}

\section{[1] MUltilingual ETHiopia}

Ethiopia is the second most populous country in Africa, with close to 90 million inhabitants - who speak approximately 90 different languages. Consequently, linguistic diversity is found not only at national and regional levels, but also in local administrative units, rural and urban speech communities, schools, institutions, and even in individual households. Any effort to establish a sustainable and holistic development in such a multilingual nation requires proper recognition and management of its socio-cultural diversity, in which language plays a key role, since it often serves as the principal indicator for the ethnic identity of individuals. As Abbink (2014) notes, however, it is not always feasible to establish an unambiguous match between linguistic and ethnic identities in multilingual contexts.

It was only in 1991 that Ethiopia adopted an ethnic federal political system, within which its current nine regional states were mainly organised along lines of ethno-linguistic identities ${ }^{1}$. Moreover, the 1994 constitution gives equal rights to all Ethiopian languages and encourages their development. Since then, Amharic - the former official language of Ethiopia - now only functions as the working language of the federal government, whereas regional governments are allowed to select their own working language for local administration. Thus, the government of Ethiopia actively promotes multilingualism and multiculturalism, the implementation of which has now been in place for the last two decades.

According to some estimates, Ethiopian Sign Language is used by nearly two million people. Most of the spoken Ethiopian languages belong to two major phyla: Afro-Asiatic and Nilo-Saharan. Among the seventeen Nilo-Saharan languages spoken in Ethiopia, Berta $\left(205,732^{2}\right)$, Gumuz $(179,348)$, Nuer $(151,195)$,

[1] Note that some of the regional states, such as the Southern Nations, Nationalities and Peoples Regional (SNNPR) state, include close to 56 ethnic groups, yet ethno-linguistic arrangements are only in place at Zonal and Wereda levels.

[2] The number of speakers mentioned in this introduction is directly taken from the results of the 2007 population census conducted by the Central Statistics Agency (CSA) published in 2008. Newer figures 
Me'en $(145,259)$ and Anuak $(88,037)$ have the largest speaker communities. Despite this, the great majority of Ethiopian languages belong to the Afro-Asiatic phylum. Three of the phylum's six language families are represented in Ethiopia, namely Semitic, Cushitic and Omotic - the last one is spoken exclusively in Ethiopia (Azeb 2013) ${ }^{3}$. According to the national census conducted in 2007, Afro-Asiatic languages with more than one million native speakers in Ethiopia are: Afan Oromo (25 mio), Somali (4,6 mio), Sidaamu Afoo ${ }^{4}(3 \mathrm{mio})$, Afar (1,3 mio) and Hadiyyisa (1,3 mio) from the Cushitic family; Wolaytta (1,5 mio) and Gamo (1,1 mio) from the Omotic family; and Amharic (21,6 mio), Tigrinya (4,3 mio) and the so-called Gurage varieties (1,6 mio) from the Ethio-Semitic family.

Beside the vibrant Ethiopian languages with millions of native and second language speakers, there are also languages that are on the verge of extinction or are already lost for good. The Ethio-Semitic Gafat is one of the languages that has disappeared with few traces. Other languages, like Ongota and Murle, are highly endangered. One language, the Ethio-Semitic Gə`əz, for which a rich written tradition has existed since ancient times, lost its native speaker community hundreds of years ago, but has been retained for liturgical purposes in the Ethiopian Orthodox Church until today. The Ethiopian Sign Language, on the other hand, which has been used for many years in specialised schools to teach deaf students, is only recently receiving more attention.

Over the last two decades, nearly half of the Ethiopian languages have been converted into written languages. In particular, the 1990s have witnessed the design and development of orthographies for a number of languages that were exclusively used for oral communication. Many of them were immediately introduced into the school system as a school subject and/or language of instruction in primary schools, cf. Moges (2010). Those languages that had been transcribed into written form before the 1990s, like Amharic or Tigrinya, are using the Ethiopic script, whereas many of the languages that were written after the 1990s opted for the Latin script ${ }^{5}$, including major languages like Afan Oromo, Sidaamu Afoo, Afar and Wolaytta, cf. Meyer (2008).

Currently, nearly 40 Ethiopian languages are used in schools for mother tongue education in different ways and at different levels. The new language

may differ, as it has been almost a decade since this survey.

[3] Notice that Ethiopian names consist of the personal name followed by the names of the father and grandfather. Following Ethiopian tradition we have chosen to use the personal names in the references in the text, and to use the personal name as the head word in the list of references. Non-Ethiopian names have surnames as the head word.

[4] In this volume, Sidaamu Afoo and Hadiyyisa are used as names of the languages in line with the names used by the native speakers.

[5] It should be pointed out that some Cushitic languages that got a writing system in the 1990's, such as Awngi and Kebena, are written with the Ethiopic script. 
policy also encourages the use of local languages in the mass media and administration. Yet the implementation of this policy requires a lot of both financial and professional capacities. Most importantly, the languages themselves must be developed to serve in new formal functions. They have to extend their terminology to express new concepts; grammars and supplementary materials for their use in education must be prepared. Furthermore, qualified personnel are needed to expand our knowledge of these languages, to assist in languagerelated challenges, and provide direction and leadership for various institutional initiatives. In short, the use of about 40 languages in different formal settings in Ethiopia today calls for constant efforts of capacity building at all levels and by all stakeholders, including universities.

\section{[2] CAPACITY BUILDING EFFORTS}

Against the backdrop of such a huge demand to build the capacity of languages and institutions, Addis Ababa University, Hawassa University, University of Oslo and the Norwegian University of Science and Technology initiated the international project Linguistic capacity building: tools for the inclusive development of Ethiopia, which is financed by the Norwegian Agency for Development Cooperation (NORAD) under its NORHED program from 2014-2018. The main aim of the project is to increase the knowledge and capacity at Ethiopian universities to develop resources for disadvantaged spoken and signed languages, so that children and adult speakers of these languages will be able to use them in education and other democratic arenas. For this purpose, the project is involved in various activities, including linguistic research, preparation of short-term training for local language specialists, development of graduate programs in linguistics and communication, $\mathrm{PhD}$ training, corpus preparation for several languages and establishing networks between stakeholders.

Within the inclusive scope of the project are diverse languages with different levels of development, for which the project's activities are tailored accordingly. The first group of languages comprises Amharic, Afan Oromo, Tigrinya and Somali. These are languages which have established themselves well in education, media and administration. Accordingly, the intervention of the project mainly focuses on corpus development and adaptation to technology. In the second group, languages like Sidaamu Afoo, Wolaytta, Hadiyyisa and Gamo are included, i.e. fairly well developed languages that are also employed in education, media and administration. Nonetheless, there is a need to strengthen these efforts by conducting research and providing expert opinion on issues of standardisation and preparation of supplementary school materials, such as dictionaries and grammar books. Thirdly, the project focuses on languages for 
which local authorities have initiated the development of orthographies and efforts are underway to use them in mother tongue education. In this group, Aari, Hamar and Gurage varieties are considered. The project assists the development of these languages by providing basic linguistic descriptions, which are crucial for designing orthographies and school materials. Moreover, it supports the development of supplementary school materials to be used in early grades and by conducting short-term trainings at local level.

Furthermore, the project gives special attention to the development of Ethiopian Sign Language, which caters for the needs of close to two million deaf or hard of hearing Ethiopians. The project works on the grammatical description of the language, trains experts, launches a graduate program and creates awareness.

\section{[3] THis VOLUME}

As part of its effort to develop the linguistic knowledge of the Ethiopian languages and deal with practical linguistic challenges, the project organised a research workshop in Rondane, Norway, 1-4 September 2015, at which original research was presented. This volume presents articles written by project participants on various aspects of the languages spoken in Ethiopia. The languages primarily covered in this volume are Amharic, Sidaamu Afoo, Hadiyyisa, Gamo, Inor, Hamar and Sezo. The topics of the contributions range from the description of specific grammatical aspects of a language to socio-political discourse; and from psycholinguistics to the issues of mother tongue education. The papers included in this volume can be generally categorised into the following major themes.

First, there are five contributions that deal with different grammatical aspects of languages. Three of these are specifically concerned with the phonology of individual languages. Baye Yimam investigates the phonological features peculiar to the Amharic variety in South Wello. He concludes that this variety does indeed constitute a distinct Amharic dialect and should be recognised as such. Tsehay Abza looks at the phonology of Inor, a peripheral Western Gurage language, with the intention of determining the phonemic status of consonants and vowels, which was a subject of contention in earlier studies. She grants phonemic status to labialised and palatalised consonants, and to the high central vowel. Shifting our attention from segmental to supra-segmental features, Girma Mengistu Desta examines in detail the tonology of Sezo. He finds that Sezo has two tonemes, i.e. high and low, and provides an in-depth description of the crucial role that tone plays in the lexicon of the language. The other two contributions deal with grammatical aspects of Hamar, a lesser-known South 
Omotic language. Binyam Sisay Mendisu describes the main aspects of negation in Hamar with some notes on linguistic typology. The paper focuses on standard negation and negative interrogatives, and addresses the effects of polarity on person agreement, as well as the marking of tense, aspect and mood. The negation of non-verbal, existential and imperative clauses is also briefly discussed. Moges Yigezu describes morphophonological aspects of Hamar. He identifies three commonly occurring morphophonological processes, namely the spreading of nasality, the spreading of place of articulation and the spreading of manner of articulation.

The second group of papers focuses on orthography and script. Ronny Meyer provides a historical and social account of the Ethiopic script by dealing with the origin, linguistic modification and socio-cultural implications of the script. Feda Negesse and Derib Ado reports on a psycholinguistic experiment concerning the visual recognition of graphic variants of the Ethiopic script as currently used in Amharic. The study notes that frequency of use is directly connected to the visual recognition of letters. The third contribution by Shimelis Mazengia addresses practical challenges of the Hadiyyisa orthography. The study reveals that students have difficulties in distinguishing phonological quantity and vowel length. The study also touches upon some of the problems students face when transferring their knowledge of Hadiyyisa orthography into learning English. At the end, the paper provides suggestions for revision, based on the principles of economy and regularity.

The third major theme which emerges from the studies collected in this volume is the issue of words and names. Lutz Edzard sheds light on the complexity of lexicographic comparison in Semitic languages, by exploring the important role that the lexicon plays in genetic classification in the context of South Arabian and Ethio-Semitic languages. He stresses that serious engagement on the applicability of lexicostatistics for genetic classification is needed. Zelealem Leyew closely investigates personal names in Hadiyya. He describes the various social, economic and political connotations of indigenous names in the Hadiyya culture, examines the linguistic features of Hadiyya names, and gives insights into the recent trend of shifting to Amharic and Biblical names.

The issue of standardisation and identity is the fourth thematic topic in this volume. Almaz Wasse Gelagay examines the standardisation of Gamo and its challenges. She finds that the standard form currently employed is based on a single dialect of Gamo known as Dače and recommends a 'dialect democracy approach' to establish a neutral standard variety. Hirut Woldemariam investigates the challenges of language planning in a context where identity is closely tied to dialectal varieties, by taking Gamo as a case in point. She recommends 
that identity planning should go hand in hand with language planning in such settings. Kjell Magne Yri explores the challenges that are faced by Sidaamu Afoo in coining specialised grammar terminology. He presents his views regarding linguistic terms which are currently in use and their negative effects for learners. He suggests using locally relevant and everyday words in terminology development.

Issues of social and political discourse are the focus of the last two papers in this volume. Nigussie Meshesha Mitike and Kjell Magne Yri provide a detailed account of three Sidaama folk media in the light of discourse analysis and speech act theory. They give a linguistic and contextual analysis of selected songs and lullabies and illustrate how the community traditionally expresses its social and political grievances via those media. Finally, Fekede Menuta and Ruth Vatvedt Fjeld examine cursing expressions in Gurage and Norwegian culture. They describe the role of cursing in each of the two cultures and conclude with a comparative note. Unlike in the Nordic culture, cursing is a highly gendered practice in Gurage, which also has a regulatory function in society.

\section{[4] THE REVIEW PROCESS}

In order to achieve the highest possible quality for the papers in this volume we have had two reviewers for each paper. No matter how good a scientific paper is to start with, it can always be improved. This is why reviewing is such an important part of the publishing process. With the help of thorough, serious reviewers the authors get the chance to see that some points are unclear to others, that certain arguments could be better focused, that background information might be added for the readers to improve understanding of the paper, and perhaps most importantly, that earlier work on the subject should not be missed out in the relevant sections.

The reviewers for this volume are experts from universities and academic institutions across the world, from Australia, Ethiopia, Finland, France, Germany, Great Britain, Kenya, Israel, Italy, Japan, Norway, Netherlands, Sweden and the USA. They have been very thorough and constructive, and a few even agreed to review more than one paper. We are very grateful for their work, and honour them by mentioning their names here. (Some reviewers wanted to stay anonymous, which we of course respect.) Their names are given in alphabetical order:

Anbessa Teferra (Tel Aviv University), Andreas Wetter (HumboldtUniversität zu Berlin), Anne Golden (University of Oslo), Avihai Shivtiel (em., University of Leeds), Azeb Amha (African Studies Centre, Leiden University), Baye Yimam (Addis Ababa University), Daniel Harbour (Queen Mary University 
of London), Elena Tkachenko (Oslo and Akershus University College of Applied Sciences), Guri Bordal Steien (University of Oslo), Jan Svennevig (University of Oslo), Janet Richmond (Edith Cowan University, Western Australia), Jun Ikeda (University of Tsukuba), Kjell Magne Yri (University of Oslo), Kristoffer Kruken (University of Oslo), Lameen Souag (Centre national de la recherche scientifique, Paris), Lutz Edzard (Friedrich-Alexander-Universität ErlangenNürnberg), Marit Julien (Lund University), Mary Pearce (SIL International), Matti Miestamo (University of Helsinki), Mauro Tosco (University of Turin), Michael R. Marlo (University of Missouri), Östen Dahl (Stockholm University), Peter Barasa (Moi University), Rolf Theil (University of Oslo), Ronny Meyer (Addis Ababa University), Stephan Guth (University of Oslo), Tore Nesset (UiT The Arctic University of Norway).

\section{AC KNOWLEDGMENTS}

This work has had financial support from the Norwegian Agency for Development Cooperation (NORAD), under its NORHED program, for the project Linguistic Capacity Building - Tools for the inclusive development of Ethiopia. It has also received support from the Centre for multilingualism in society across the lifespan (MultiLing) at the University of Oslo, funded by the Research Council of Norway, project no. 2232 65. Our colleagues, linguists as well as administrators, have been central to much of the work presented here. We mention the Text Laboratory, MultiLing and the administration at Department of Linguistics and Nordic Studies at the University of Oslo and Department of Linguistics at Addis Ababa University, and of course all the project participants (staff and PhD students from Norway and Ethiopia). We also thank Sarah Whitehead for her thorough proofreading. Finally, Kristin Hagen deserves high praise for the efforts and time she has devoted to the technical work on this book.

\section{REFERENCES}

Abbinks, Jon. 2014. Language and Peoples in Ethiopia and Eritrea. In Siegbert Uhlig (ed.), Encyclopedia Aethiopica, vol. 5: Y-Z, 381-388. Wiesbaden: Harrassowitz.

Azeb Amha. 2013. Omotic. In Zygmunt Frajzyngier (ed.), The Afroasiatic Languages, 423-504. Cambridge: Cambridge University Press.

CSA. 2008. Summary and Statistical Report of the 2007 Population and Housing Census: Population Size by Age and Sex. Addis Ababa: Population Census Commission.

Meyer, Ronny. 2008. Sudan and the Horn of Africa. In Ulrich Ammon, Nobert Dittmar, Klaus J. Mattheier \& Peter Trudgill (eds.), Sociolinguistics: an Interna- 
tional Handbook of the Science of Language and Society, vol. 3, 1951-1956. Berlin: Walter de Gruyter.

Moges Yigezu. 2010. Language Ideologies and Challenges of Multilingual Education in Ethiopia: the case of Harari Region. Addis Ababa: OSSREA.

CONTACTS

Binyam Sisay Mendisu

Department of Linguistics, Addis Ababa University

b.s.mend@gmail.com

Janne Bondi Johannessen

Text Laboratory and MultiLing, University of Oslo

jannebj@iln.uio.no 